\title{
Ceftaroline in complicated skin and skin-structure infections
}

This article was published in the following Dove Press journal:

Infection and Drug Resistance

18 January 2012

Number of times this article has been viewed

\author{
Paul O Hernandez' \\ Sergio Lema ${ }^{2}$ \\ Stephen K Tyring ${ }^{3}$ \\ Natalia Mendoza ${ }^{2,4}$ \\ 'University of Texas School \\ of Medicine at San Antonio, \\ San Antonio, TX, ${ }^{2}$ Woodhull \\ Medical and Mental Health Center, \\ Brooklyn, NY, ${ }^{3}$ Department of \\ Dermatology, University of Texas \\ Health Science Center at Houston, \\ Houston, TX, USA; ${ }^{4}$ Department of \\ Dermatology, El Bosque University, \\ Bogotá, Colombia
}

Correspondence: Natalia Mendoza Department of Dermatology, El Bosque University, Woodhull Medical and Mental Health Center, 37 Wall Street, 5L, New York, NY, USA 10005

Tel +l 8326309350

$\mathrm{Fax}+\mathrm{I} 7135288848$

Email natyt99@yahoo.com

\begin{abstract}
Ceftaroline is an advanced-generation cephalosporin antibiotic recently approved by the US Food and Drug Administration for the treatment of complicated skin and skin-structure infections (cSSSIs). This intravenous broad-spectrum antibiotic exerts potent bactericidal activity by inhibiting bacterial cell wall synthesis. A high affinity for the penicillin-binding protein $2 \mathrm{a}$ (PBP2a) of methicillin-resistant Staphylococcus aureus (MRSA) makes the drug especially beneficial to patients with MRSA cSSSIs. Ceftaroline has proved in multiple well-conducted clinical trials to have an excellent safety and efficacy profile. In adjusted doses it is also recommended for patients with renal or hepatic impairment. Furthermore, the clinical effectiveness and high cure rate demonstrated by ceftaroline in cSSSIs, including those caused by MRSA and other multidrug-resistant strains, warrants its consideration as a first-line treatment option for cSSSIs. This article reviews ceftaroline and its pharmacology, efficacy, and safety data to further elucidate its role in the treatment of cSSSIs.
\end{abstract}

Keywords: ceftaroline, cephalosporin, complicated skin and skin-structure infections, cSSSIs, MRSA, Teflaro ${ }^{\circledR}$

\section{Overview of complicated skin and skin-structure infections}

Complicated skin and skin-structure infections (cSSSIs) are severe dermatologic infections either involving deep soft tissue (fascia and/or muscle layers), requiring significant surgical intervention, or existing in combination with significant underlying disease that complicates the response to treatment. ${ }^{1}$ The US Food and Drug Administration (FDA) recently termed these infections "acute bacterial skin and skin-structure infections" (ABSSSIs) based on their predominant bacterial etiology. ${ }^{2}$ Other national or international classification and nomenclature groups often refer to these infections as complicated skin and soft tissue infections (cSSTIs).,

\section{Microbiology of cSSSls}

The microbiologic causes of cSSSIs are diverse, with aerobic Gram-positive cocci most commonly isolated from these infections, specifically Staphylococcus aureus. Gram-negative bacilli, anaerobic bacteria, and other mixed microorganisms may also be involved (Table 1). ${ }^{5-15}$ In the US, studies have reported methicillin-resistant S. aureus (MRSA) as the leading cause of cSSSIs, with MRSA isolated in almost $60 \%$ of cases. ${ }^{16,17}$ Community-acquired (CA) cSSSIs usually involve infection with S. aureus, Streptococcus pyogenes, or enterococci. One US study reported the MRSA USA300 clone as the predominant strain causing CA-MRSA cSSSIs. ${ }^{18} \mathrm{~A}$ recent 
Table I Most common bacterial isolates identified in $\mathrm{cSSSI}^{5-15}$

\begin{tabular}{ll}
\hline Gram-positive & Gram-negative \\
\hline Staphylococcus aureus: MSSA, MRSA & Escherichia coli \\
Streptococcus pyogenes & Pseudomonas aeruginosa \\
Enterococcus faecalis & Klebsiella pneumoniae \\
Enterococcus faecium & Proteus mirabilis \\
Coagulase-negative staphylococci & Klebsiella oxytoca \\
Streptococcus agalactiae & Enterobacteriaceae \\
Peptostreptococcus spP & \\
\hline
\end{tabular}

Abbreviations: MSSA, methicillin-susceptible Staphylococcus aureus; MRSA, methicillinresistant Staphylococcus aureus.

Japanese study indicating that overuse of over-the-counter triple antibiotic creams in the treatment of minor cuts and scrapes may influence the emergence of MRSA USA300 raises significant concerns. ${ }^{19}$ Hospital-acquired (HA) cSSSIs usually involve infection with $S$. aureus, Pseudomonas aeruginosa, enterococci, Escherichia coli, and other Enterobacteriaceae, with increased prevalence of MRSA observed in hospitals as well as the community. ${ }^{8,11,12}$

\section{Management of cSSSIs}

Although cSSSIs arise in both communities and hospitals, they generally require initial and definitive treatment in a hospital setting due to their severity and potential for serious complications. A US hospital-based study reported secondary bacteremia in $47.8 \%$ of patients with cSSSIs, resulting in a higher mortality rate among bacteremic patients than nonbacteremic patients (7.9\% vs $1.0 \%$, respectively). ${ }^{20}$ Bacteremia in cSSSIs is also associated with an almost fourfold increase in mortality rate when initial antibiotic therapy fails in comparison to successful initial antibiotic therapy ( $1.7 \%$ vs $0.5 \%$, respectively) and increased length of hospital stay and costs..$^{20-22}$

Definitive antimicrobial therapy is pathogen-specific based on culture results, antimicrobial susceptibility, and the clinical response of the infection to empirical therapy. Rapidly advancing infections constitute the need for surgical exploration with histopathological examination to identify necrotizing processes. If severe, rapidly advancing infections may require extensive debridement and alterations to subsequent antimicrobial therapy. ${ }^{5}$

Limited antimicrobial agents are available for the treatment of cSSSIs due to a combination of the increasing prevalence of antimicrobial resistance in bacteria and a diminished industry focus on antimicrobial drug research and development; ${ }^{23}$ thus, successful treatment with approved antimicrobial compounds remains a challenge. Because of the high prevalence of MRSA in skin and soft tissue infections presenting to hospitals, empirical therapy should include MRSA coverage. ${ }^{16}$ Suspicion of infection with MRSA is based on the following criteria: (1) whether the infection is CA or HA, (2) individual risk factors for MRSA, and (3) severity of the infection. In order to improve treatment outcomes in $S$. aureus infections, surgical drainage and debridement, wound culture, and prompt initiation of appropriate empirical antimicrobial therapy is recommended. ${ }^{24,25}$ Definitive antimicrobial therapy in an MRSA infection is dependent on whether the infection is caused by CA-MRSA or HA-MRSA (Table 2). ${ }^{25}$

In February 2011, the Infectious Diseases Society of America (IDSA) published guidelines for the treatment of hospitalized adults and children with cSSTIs (synonymous with our definition of cSSSIs). The IDSA recommendation for adults includes empirical therapy with MRSA coverage and culturing for susceptibility data, in addition to surgical debridement and broad-spectrum antibiotics. Antibiotics used in empirical therapy include intravenous (IV) vancomycin, daptomycin, telavancin, linezolid, and clindamycin. Oral linezolid and oral clindamycin are also empirical therapy options.

In hospitalized children with cSSSIs, the IDSA recommends vancomycin. If the child is stable, nonbacteremic, and resistance to clindamycin is low, empirical intravenous clindamycin is an option. A transition to oral clindamycin therapy is indicated if the strain is susceptible to clindamycin. Intravenous or oral linezolid are also therapeutic alternatives for children. ${ }^{4}$

FDA-approved parenteral antimicrobial agents for the treatment of ABSSSIs (synonymous with our definition of cSSSIs) with anti-MRSA activity include vancomycin, quinupristin/dalfopristin, linezolid, daptomycin, telavancin, tigecycline, and ceftaroline. ${ }^{26}$ Vancomycin continues to be the first-line treatment for MRSA infections, as no other

Table 2 Recommended treatment for community-acquired MRSA versus hospital-acquired MRSA infection (including cSSSIs) 4,24,25

\begin{tabular}{ll}
\hline CA-MRSA & HA-MRSA \\
\hline Usually not multidrug resistant & Usually multidrug resistant \\
Susceptibility testing: & Susceptibility testing: \\
trimethoprim-sulfamethoxazole & vancomycin, rifampin, \\
(TMP-SMX), clindamycin, vancomycin & linezolid \\
Treatment: TMP-SMX, & Treatment: vancomycin, \\
clindamycin, ciprofloxacin, & linezolid, daptomycin, \\
gentamicin, ${ }^{a}$ vancomycin, linezolid, & tigecycline, or rifampin plus \\
daptomycin, or telavancin & fusidic acid (where available) \\
\hline
\end{tabular}

Note: anly in combination with other agents.

Abbreviations: MRSA, methicillin-resistant Staphylococcus aureus; TMP-SMX, trimethoprim-sulfamethoxazole. 
antimicrobial agent has proved to have superior efficacy. ${ }^{26-28}$ Fusidic acid is marketed for the treatment of staphylococcal infections in at least 23 countries. Countries such as Canada, the UK, Ireland, and New Zealand have approved fusidic acid for use in the treatment of skin infections, including those caused by MRSA. ${ }^{29}$ A US multicenter Phase II, randomized, double-blind clinical trial comparing the efficacy and safety of an oral fusidic acid loading-dose regimen to oral linezolid in the treatment cSSSIs was conducted between August 2009 and March 2010. The results of the study indicated fusidic acid had similar efficacy, safety, and tolerability as linezolid in the treatment of cSSSIs. This study demonstrates an attempt to introduce fusidic acid into the US market, pending additional Phase III clinical trials and FDA approval. ${ }^{30}$

\section{Issues of resistance}

The major pathogen of concern is MRSA due to its high prevalence of infection and multidrug-resistance. ${ }^{31-33}$ US surveillance data over a 12-year period between 1992 and 2004 indicated a 59.5\% rate of methicillin resistance among $S$. aureus nosocomial infections in intensive care unit patients. ${ }^{34}$ MRSA has also become more common in infections in long-term care facility patients and in CA infections. ${ }^{6,35,36}$ The most commonly isolated MRSA clone in the US is MRSA USA300, identified in $99 \%$ of CA-MRSA isolates in one study. ${ }^{18}$ The spread of MRSA USA300 has been reported around the world. Studies in Austria and Canada reported identification of MRSA USA300 in CA-MRSA isolates (2.2\% and $73.7 \%$, respectively). ${ }^{33,37}$

In the US, there has been a rapid and alarming increase in CA-MRSA isolated from infections. US surveillance data over a 10-year period between 1998 and 2007 indicated a $387.6 \%$ increase in CA-MRSA infection, rising from $7.3 \%$ in 1998 to $35.6 \%$ in $2007 .{ }^{32}$ Conversely, there has been a $27.9 \%$ decrease in HA-MRSA infection in the US over the same 10-year period, falling from $25.4 \%$ in 1998 to $18.3 \%$ in $2007 .{ }^{32}$ Studies in the UK indicate the incidence of CA-MRSA infection is very low $(<1 \%)$, although incidence does appear to be on the rise. ${ }^{38}$ Moreover, CA-MRSA has also been isolated from healthy individuals. One study in India found that 166 of 1000 (16.6\%) healthy participants were carriers of CA-MRSA in their anterior nares. ${ }^{39}$

Consistent with US surveillance data between 1998 and 2007, the Centers for Disease Control and Prevention (CDC) reported a $28 \%$ decrease of invasive MRSA infection in healthcare settings between 2005 and 2008. The CDC also reports a $17 \%$ decrease in CA-MRSA infection in people with recent exposure to health care settings. As such, the
CDC recognizes the continued need to address this public health concern. In contrast, the $\mathrm{CDC}$ reported that CA-MRSA infection is increasing rapidly in the community. ${ }^{40}$

Resistance to vancomycin has also been reported, in vancomycin-resistant enterococci (VRE) and the more recently emergent vancomycin-resistant $S$. aureus (VRSA). ${ }^{27,41,42}$ Enterococci demonstrate one of the highest rates of vancomycin resistance. A US study conducted between 2007 and 2008 of isolates collected from hospitalized patients reported vancomycin resistance rates among Enterococcus faecalis and Enterococcus faecium were $5.4 \%$ and $75.4 \%$, respectively. ${ }^{41}$ In Europe, a $5.1 \%$ rate of VRE was reported across 33 medical centers. ${ }^{43}$ A study in India reported a $1.4 \%$ rate of vancomycin resistance in $S$. aureus isolates collected from healthy individuals. ${ }^{39}$

Decreased susceptibility to vancomycin has also been observed in vancomycin-susceptible $S$. aureus (VSSA). In the presence of selection pressure, VSSA isolates are able to transform their cell wall to become less susceptible to vancomycin, termed vancomycin-intermediate $S$. aureus (VISA). VISA isolates may progress through a precursor phenotype known as heteroresistant vancomycin-intermediate $S$. aureus (hVISA). A systematic review and meta-analysis evaluating the significance of hVISA isolates throughout the world reported that overall prevalence remains low at $1.3 \%$ of all MRSA isolates tested. ${ }^{44}$

Resistance to antimicrobial agents used in the treatment of cSSSIs is not limited to methicillin and vancomycin; there is also evidence of resistance to linezolid, daptomycin, streptogramins, ertapenem, fluoroquinolones, and glycopeptide antibiotics. ${ }^{28,31}$ In order to decrease resistance and cross-resistance development, optimizing antibiotic use through strict prescription habits as well as strict use of overthe-counter topical antibiotic creams is key. The increasing prevalence of resistance to existing antimicrobial agents underscores not only the importance of judicious antibiotic use by clinicians, but also the need for the development of new antibiotics such as ceftaroline, an emerging cephalosporin that recently gained regulatory approval in the US for the treatment of cSSSIs. ${ }^{23,45}$

\section{Ceftaroline}

Ceftaroline fosamil (Teflaro ${ }^{\circledR}$; Forest Laboratories, Inc, New York, NY) is a novel advanced-generation cephalosporin with broad-spectrum activity against Gram-positive, many Gram-negative, some anaerobic, and multidrug-resistant strains that cause serious CA and HA pneumonia and skin and skin-structure infections (Table 3) ${ }^{46-55}$ Ceftaroline is the 
Table 3 In vitro activity of ceftaroline against common Grampositive and Gram-negative bacterial isolates ${ }^{47,52,80,81}$

\begin{tabular}{lc}
\hline Gram-positive & Gram-negative \\
\hline Staphyloccocus aureus & Enterobacteriaceae \\
MSSA & Escherichia coli \\
MRSA & Ceftazidime-susceptible \\
VISA & ESBL-positive E. colia \\
Linezolid resistant & Klebsiella pneumoniae \\
Coagulase-negative staphylococci & Ceftazidime-susceptible \\
Streptococcus pyogenes & ESBL-positive K. pneumoniae ${ }^{\mathrm{a}}$ \\
Streptococcus agalactiae & Enterobacter cloacae \\
Enterococcus faecalis & Proteus mirabilis \\
Vancomycin-susceptible & Citrobacter freundii \\
Vancomycin-resistant & Morganella morganii \\
Streptococcus pneumoniae & Serratia marcescens \\
Penicillin susceptible & Shigella spp \\
Penicillin intermediate & Moraxella catarrhalis \\
Penicillin resistant & Haemophilus influenzae \\
Levofloxacin resistant & $\beta$-lactamase positive, ampicillin \\
& resistant \\
Multidrug resistant & $\beta$-lactamase negative, ampicillin \\
& resistant \\
Viridans group streptococci & Pasteurella multocida \\
& Pseudomonas aeruginosa ${ }^{\mathrm{b}}$ \\
\hline
\end{tabular}

Notes: ${ }^{\text {aC }}$ Conflicting data for activity against ESBL-positive $E$ coli and ESBL-positive Klebsiella pneumoniae, ${ }^{79-81}$ b conflicting data for activity against $P$. aeruginosa: in vitro studies show only limited activity whereas clinical studies show a high clinical cure rate. ${ }^{15,79-81}$

Abbreviations: MSSA, methicillin-susceptible Staphylococcus aureus; MRSA, methicillinresistant Staphylococcus aureus; VISA, vancomycin-intermediate Staphylococcus aureus; ESBL, extended-spectrum $\beta$-lactamase.

active metabolite of its prodrug form, ceftaroline fosamil (herein referred to as ceftaroline). In several clinical studies, ceftaroline demonstrated potent efficacy against common pathogens implicated in cSSSIs, including MRSA and $\beta$-hemolytic streptococci. ${ }^{10,14,15,56,57}$ In October 2010, the FDA approved ceftaroline for the treatment of CA bacterial pneumonia and cSSSIs, including MRSA-associated infections (Table 4). ${ }^{45}$

\section{Mechanism of action}

Ceftaroline is a bactericidal $\beta$-lactam antibiotic that targets and binds penicillin-binding proteins (PBPs) to inhibit cell wall synthesis through interference of peptidoglycan cross-linking. ${ }^{58}$ It binds with especially high affinity to the MRSA-associated PBP2a. ${ }^{59,60}$ Ceftaroline also binds with high affinity to the PBP2x possessed by penicillin-resistant Streptococcus pneumoniae (PRSP). Specifically, this binding inhibits transpeptidase or transglycosidase bacterial enzymes necessary for cell wall synthesis, thereby exerting its bactericidal effect. ${ }^{59}$ The 1,3-thiazole ring in ceftaroline's molecular structure confers its anti-MRSA activity. ${ }^{61,62}$ The 1,2,4-thiadiazole ring confers Gram-negative penetration and increased affinity for transpeptidase enzyme..$^{59,62}$
Table 4 Ceftaroline ${ }^{\mathrm{a}}$ in the treatment of cSSSIs in the US

\begin{tabular}{|c|c|}
\hline \multirow[t]{2}{*}{ Dosage form } & Injection; powder for reconstitution \\
\hline & Stable in: I/2 NS, D5W, LR, NS \\
\hline Usual dosage range & $600 \mathrm{mg}$ IV every 12 hours for $5-14$ days \\
\hline Administration & Slow IV infusion over 60 minutes \\
\hline Dosing for renal & $\mathrm{CL}_{\mathrm{Cr}} 3 \mathrm{I}-50 \mathrm{~mL} /$ minute: administer $400 \mathrm{mg}$ \\
\hline \multirow[t]{5}{*}{ impairment } & every 12 hours $\mathrm{CL}_{\mathrm{Cr}} 15-30 \mathrm{~mL} /$ minute: \\
\hline & administer $300 \mathrm{mg}$ every 12 hours \\
\hline & $\begin{array}{l}\mathrm{CL}_{\mathrm{Cr}}<15 \mathrm{~mL} / \text { minute and ESRD patients } \\
\text { receiving hemodialysis: administer }\end{array}$ \\
\hline & 200 mg every 12 hours; should be given \\
\hline & after hemodialysis, if applicable \\
\hline Approved use & Treatment of acute cSSSIs: caused by \\
\hline & susceptible isolates of Staphylococcus \\
\hline & aureus (MSSA and MRSA), Streptococcus \\
\hline & pyogenes, Streptococcus agalactiae, \\
\hline & Escherichia coli, Klebsiella pneumoniae, \\
\hline & and Klebsiella oxytoca \\
\hline
\end{tabular}

Notes: aAdvanced-generation cephalosporin antibiotic; bFDA-approved October 2010. Abbreviations: NS, normal saline; D5W, $5 \%$ dextrose in water; LR, lactated Ringer's solution; IV, intravenous; $\mathrm{CL}_{\mathrm{Cr}}$, creatinine clearance; ESRD, end-stage renal disease; MSSA, methicillin-susceptible Staphylococcus aureus; MRSA, methicillinresistant Staphylococcus aureus.

The phosphono group, only present in the prodrug, increases its solubility. ${ }^{59,62,63}$

\section{Pharmacokinetics}

Following parenteral administration, the water-soluble prodrug is hydrolyzed to its active metabolite, ceftaroline, by plasma phosphatases. It has a volume of distribution $\left(\mathrm{V}_{\mathrm{d}}\right)$ of $28.3 \mathrm{~L}$ into the total body water compartment. ${ }^{62}$ On average, $20 \%$ of ceftaroline circulates bound to plasma proteins. It has a half-life of 2.6 hours. ${ }^{45}$

Ceftaroline mainly undergoes hydrolytic metabolism in the plasma. When incubated with pooled human liver microsomes, ceftaroline was metabolically stable, indicating that it is not a substrate for CYP450; therefore, there is minimal hepatic metabolism. ${ }^{64}$ The majority (about $88 \%$ ) of the administered dose is eliminated from the body by renal glomerular filtration, followed by fecal excretion (about $6 \%$ ), within 48 hours. ${ }^{63-65}$

Approximately half of the excreted drug is in active form, with a small quantity in inactive form (ceftaroline-M-1). ${ }^{62}$ No prodrug has been detected in the urine. This finding led to the conclusion of absolute biotransformation of the prodrug into its active metabolite, ceftaroline. ${ }^{62,65} \mathrm{In}$ a singledose administration, ceftaroline has a renal clearance of $95.6 \mathrm{~mL} /$ minute; administered in multiple doses, its renal clearance is $86.7 \mathrm{~mL} /$ minute. $^{62}$

A number of studies in healthy adults demonstrated the absorption of ceftaroline when administered either 
intravenously (IV) or intramuscularly (IM) in single or multiple doses. ${ }^{62}$ In single-dose studies, which administered ceftaroline $(250,500,750$, or $1000 \mathrm{mg})$ IV over 60 minutes, the maximum plasma concentration $\left(\mathrm{C}_{\max }\right)$ increased approximately in proportion to drug dose, 9.9, 16.5, 23.0, and $30.2 \mu \mathrm{g} / \mathrm{mL}$, respectively. ${ }^{62}$ In multiple-dose studies, there was no accumulation of the drug when 300,600 , or $800 \mathrm{mg}$ doses were administered at intervals of either 12 or 24 hours over the course of 7-14 days. ${ }^{62}$ In studies conducted by Riccobene and colleagues comparing the pharmacokinetics of a single $600 \mathrm{mg}$ IM dose to a single $600 \mathrm{mg}$ IV dose, the $\mathrm{C}_{\max }$ attained were $8.5 \mu \mathrm{g} / \mathrm{mL}$ and $19.7 \mu \mathrm{g} / \mathrm{mL}$, respectively. The time to reach maximum plasma concentration $\left(\mathrm{t}_{\text {max }}\right)$ for IM administration was 2 hours; the $t_{\max }$ for IV administration was only 0.98 hours. ${ }^{66}$

Studies by Ge and colleagues evaluated the similarities and differences in the pharmacokinetics of ceftaroline in individuals with normal renal function versus those with mild or moderate renal impairment during administration of ceftaroline $600 \mathrm{mg}$ IV (Table 5). ${ }^{62,67} \mathrm{C}_{\max }$ and $\mathrm{t}_{\max } \mathrm{did}$ not differ significantly among the three groups, whereas there was an increase in the area under the drug plasma concentration curve (AUC) and half-life $\left(t_{1 / 2}\right)$ in both the mild and moderate renal impairment groups in comparison to the normal renal function group. Furthermore, the renal clearance of ceftaroline decreased significantly in patients with mild and moderate renal impairment $(30.8 \mathrm{~mL} /$ minute and $19.3 \mathrm{~mL} /$ minute, respectively) in comparison to patients with normal renal function $(54.6 \mathrm{~mL} /$ minute $){ }^{62}$

Another study by Riccobene and colleagues investigated the pharmacokinetics of ceftaroline in patients with end-stage renal disease. ${ }^{68}$ A $400 \mathrm{mg}$ IV dose was administered over the course of 1 hour in two patient groups, one group before and one group after dialysis. Administering the dose prior to dialysis resulted in a $\mathrm{C}_{\max }$ similar to that of patients with normal renal function; however, administering the dose after dialysis resulted in a $74 \%$ increase in $\mathrm{C}_{\max }$ in comparison to patients with normal renal function. The AUC increased by $89 \%$ when ceftaroline was administered prior to dialysis and increased by $167 \%$ when administered after dialysis in comparison to patients with normal renal function. The $t_{1 / 2}$ also increased by $123 \%$ in both patient groups. ${ }^{68}$

In light of this clinical data, ceftaroline doses should be adjusted for patients with moderate renal impairment and those with end-stage renal disease. ${ }^{62,63,65,68} \mathrm{Ge}$ and colleagues recommend ceftaroline $600 \mathrm{mg}$ IV be infused over 1 hour every 12 hours for patients with normal renal function and those with mild renal impairment; for patients with moderate renal impairment the dose should be reduced to $400 \mathrm{mg} \mathrm{IV.}{ }^{61,62,67}$ The FDA recommends $400 \mathrm{mg}$ IV infused over 60 minutes every 12 hours for patients with mild renal impairment, $300 \mathrm{mg}$ IV for patients with moderate renal impairment, and $200 \mathrm{mg}$ IV for patients with end-stage renal disease, including those undergoing hemodialysis (Table 4). ${ }^{45}$

\section{Pharmacodynamics}

A mouse model study of thigh and lung infection conducted by Andes and Craig determined the pharmacokineticpharmacodynamic (PK-PD) index to predict the efficacy of ceftaroline against MRSA and Gram-negative bacilli. ${ }^{69}$ The percentage of time the serum concentrations were above the minimum inhibitory concentration $(\% \mathrm{~T}>\mathrm{MIC})$ was the PK-PD that best correlated with drug efficacy. ${ }^{59}$ The MIC is the lowest concentration that will inhibit visible growth of bacteria after overnight incubation. A lower MIC indicates a more efficacious antimicrobial agent. ${ }^{70}$ Andes and Craig reported a mean $\% \mathrm{~T}>$ MIC of $39 \%$ for $S$. pneumoniae, $21 \%$ for MRSA, and 28\% for E. coli and Klebsiella pneumoniae combined to achieve a bacteriostatic effect. A dose of $64.1 \mathrm{mg} / \mathrm{kg} / 24$ hours and a $\% \mathrm{~T}>\mathrm{MIC}$ of $50 \%$ was necessary to achieve a $2-\log _{10}$ reduction against MRSA. ${ }^{69}$

An in vitro study using a PK-PD model evaluated ceftaroline $600 \mathrm{mg}$ MRSA and hVISA activity every 8 hours and 12 hours in comparison to vancomycin $1000 \mathrm{mg}$ activity every 12 hours over a 72-hour period..$^{71}$ Ceftaroline proved superior to vancomycin against all isolates, except one to which it was equivalent; no emergent drug-resistant isolates were observed. ${ }^{71}$ Further, in a rabbit endocarditis model study, ceftaroline demonstrated a high bactericidal effect,

Table 5 Pharmacokinetics in patients with renal impairment: single-dose ceftaroline $600 \mathrm{mg} I \mathrm{~V}^{67}$

\begin{tabular}{|c|c|c|c|c|c|}
\hline $\begin{array}{l}\text { Renal status } \\
\text { (CL }{ }_{C r} \text { mL/minute) }\end{array}$ & $\begin{array}{l}t_{1 / 2} \\
\text { (hours) }\end{array}$ & $\begin{array}{l}C_{\max } \\
(\mu g / m L)\end{array}$ & $\begin{array}{l}t_{\max } \\
\text { (hours) }\end{array}$ & $\begin{array}{l}\text { AUC } \\
(\mu \mathrm{g} \cdot \text { hour } / \mathrm{mL})\end{array}$ & $\begin{array}{l}C_{R} \\
\text { (mL/minute) }\end{array}$ \\
\hline Normal $\left(\mathrm{CL}_{\mathrm{Cr}}>80\right)$ & 2.84 & 27.6 & 0.97 & 35.6 & 54.6 \\
\hline Mild $\left(\mathrm{CL}_{\mathrm{Cr}}>50-80\right)$ & 3.61 & 27.7 & 0.99 & 89.4 & 30.8 \\
\hline Moderate $\left(\mathrm{CL}_{\mathrm{Cr}}>30-50\right)$ & 4.49 & 30.5 & I.I & 114 & 19.3 \\
\hline
\end{tabular}

Abbreviations: $\mathrm{CL}_{\mathrm{Cr}}$, creatinine clearance; $\mathrm{t}_{1 / 2}$, half-life; $\mathrm{C}_{\max }$, maximum plasma concentration; $\mathrm{t}_{\max }$, time to maximum plasma concentration; $\mathrm{AUC}$, area under the concentration-time curve; $C L_{R}$, renal clearance. 
significant killing of bacteria in aortic vegetations, and superior bactericidal activity against hVISA compared to vancomycin. ${ }^{49}$ Treatment of the infected vegetations with ceftaroline $10 \mathrm{mg} / \mathrm{kg}$ IV every 12 hours resulted in $90 \%$ sterile vegetations after 4 days in comparison to vancomycin constant IV infusion, which resulted in $67 \%$ sterile vegetations. ${ }^{49}$

\section{Clinical trials of ceftaroline in cSSSIs Efficacy}

The efficacy of ceftaroline against bacteria that cause cSSSIs is well established in multiple clinical studies, one Phase II and two identical Phase III trials. A Phase II trial consisting of a total of 100 patients evaluated ceftaroline versus standard therapy (defined as vancomycin with or without adjunctive aztreonam in the study) in the treatment of cSSSIs (67 patients vs 33 patients, respectively). Ceftaroline was administered at a dose of $600 \mathrm{mg}$ IV every 12 hours for 7-14 days, and vancomycin was administered at a dose of $1 \mathrm{~g}$ IV every 12 hours with or without adjunctive aztreonam at a dose of $1 \mathrm{~g}$ IV every 8 hours for 7-14 days. There was a total of 88 patients in the clinically evaluable (CE) population. Clinical cure rates for ceftaroline in $\mathrm{CE}$ patients at test-of-cure (TOC) and end-of-therapy (EOT) visits were high (96.7\% and $98.4 \%$, respectively). ${ }^{54}$ Clinical cure rates for standard therapy in CE patients at TOC and EOT visits were lower in both instances $(88.9 \%$ and $96.3 \%$, respectively). Among the microbiologically evaluable (ME) patient population (ie, clinically evaluable and having had at least one susceptible pathogen isolated at baseline), ceftaroline demonstrated a higher microbiological success rate than standard therapy (95.2\% vs $85.7 \%$, respectively). ${ }^{54}$ The Phase II trial included adults diagnosed with skin and skin-structure infections if the infection involved deep soft tissue, required significant surgical intervention, or the infection had developed in a lower extremity in a patient with diabetes mellitus or peripheral vascular disease. ${ }^{54}$ The TOC assessments were conducted 8-14 days following the last administered dose. The mean length of therapy was similar for both the ceftaroline and standard therapy groups (7.8 days and 8.0 days, respectively). ${ }^{54}$

Two identical Phase III, multicenter, international, randomized, double-blind clinical trials (CANVAS 1 and CANVAS 2) evaluated ceftaroline versus vancomycin plus aztreonam in the treatment of cSSSIs (Table 6). ${ }^{10,15}$ Combining CANVAS 1 and 2 data, 701 patients were administered ceftaroline $600 \mathrm{mg}$ IV every 12 hours for 5-14 days and 695 patients were administered vancomycin $1 \mathrm{~g}$ IV plus aztreonam $1 \mathrm{~g}$ IV every 12 hours for 5-14 days. Both Phase III trials included patients $\geq 18$ years of age with a diagnosis of cSSSI that required $\geq 5$ days of IV antibiotic therapy. The primary study outcome was clinical cure, defined as total resolution of all signs and symptoms of baseline infection or improvement such that no further antimicrobial treatment was needed at the TOC visit (8-15 days after the last administered dose). ${ }^{10,15}$ The mean length of therapy for CANVAS 1 and 2 was similar (7 days and 6.5 days, respectively). ${ }^{10,15}$

The CANVAS 1 and 2 trials demonstrated similar cure rates for ceftaroline and vancomycin plus aztreonam in the $\mathrm{CE}$ and ME populations, as well as the modified intent-to-treat (MITT) population, which consisted of patients who received any amount of study medication. In CANVAS 1 , clinical cure rates were similar for ceftaroline and vancomycin plus aztreonam in the CE population $(91.1 \% \mathrm{vs}$ 93.3\%, respectively), ME population (92.2\% vs $94.7 \%$, respectively), and the MITT population (86.6\% vs $85.6 \%$, respectively). In CANVAS 2, clinical cure rates were also similar for ceftaroline and vancomycin plus aztreonam in the CE population (92.2\% vs $92.1 \%$, respectively), ME population (93.3\% vs $94.1 \%$, respectively), and the MITT population ( $85.1 \%$ vs $85.5 \%$, respectively) (Table 6 ).

Combined data from CANVAS 1 and 2 show a similar cure rate in $\mathrm{CE}$ patients with diabetes mellitus or peripheral vascular disease treated with ceftaroline versus vancomycin plus aztreonam (86.7\%-91.1\% and 87.2\%-93.4\%, respectively).

Table 6 Clinical cure rates by population in Phase III CANVAS I and 2 trials ${ }^{10,15}$

\begin{tabular}{|c|c|c|c|c|}
\hline & \multicolumn{2}{|l|}{ CANVAS I } & \multicolumn{2}{|l|}{ CANVAS 2} \\
\hline & \multicolumn{2}{|c|}{ Clinical cure rate \% } & \multicolumn{2}{|c|}{ Clinical cure rate \% } \\
\hline & Ceftaroline $^{a}$ & Vancomycin plus aztreonam $^{b}$ & Ceftaroline $^{a}$ & Vancomycin plus aztreonam \\
\hline \multicolumn{5}{|c|}{ Population } \\
\hline CE & 91.1 & 93.3 & 92.2 & 92.1 \\
\hline ME & 92.2 & 94.7 & 93.3 & 94.1 \\
\hline MITT & 86.6 & 85.6 & 85.1 & 85.5 \\
\hline
\end{tabular}

Notes: ${ }^{2} 600 \mathrm{mg}$ of ceftaroline intravenously every 12 hours for 5-14 days; ${ }^{b} \mathrm{I} g$ of vancomycin plus I $\mathrm{g}$ of aztreonam intravenously every 12 hours for 5-14 days. Abbreviations: CANVAS, ceftaroline versus vancomycin in skin and skin structure infections; MITT, modified intent-to-treat; CE, clinically evaluable; ME, microbiologically evaluable. 
The clinical cure rate was higher in CE patients with a lower extremity infection and either diabetes mellitus or peripheral vascular disease treated with ceftaroline in comparison to vancomycin plus aztreonam (100\% and $81.8 \%-94.7 \%$, respectively)..$^{10,15}$

The CANVAS trials demonstrated similar clinical cure rates for cSSSIs specifically caused by MRSA. In CANVAS 1, clinical cure rates for MRSA cSSSIs in the ceftaroline and vancomycin plus aztreonam groups were $95.1 \%$ and $95.2 \%$, respectively; in CANVAS 2, they were $91.4 \%$ and $93.3 \%$, respectively. Ceftaroline clinical cure rates by specific pathogen involved are reported in Table 7. It should be noted that ceftaroline demonstrated a $71.4 \%$ clinical cure rate against $P$. aeruginosa in the ME population in CANVAS 2 but did not show any activity against $P$. aeruginos $a$ in either the Phase II or CANVAS 1 trials. ${ }^{15}$

Ceftaroline $\mathrm{MIC}_{90}$ values, the lowest concentration at which $90 \%$ of the bacteria are inhibited, for the most common pathogens encountered in cSSSIs are reported in Table $8 .^{10,14,15}$ In CANVAS 1 and 2, the most common pathogen was $S$. aureus, which appeared in $75 \%$ and $82 \%$ of isolates, respectively. Of these $S$. aureus isolates, MRSA was identified in $43 \%$ and $30 \%$, respectively. ${ }^{10,15}$ S. aureus isolates were susceptible to ceftaroline at a lower $\mathrm{MIC}_{90}(\leq 0.5 \mathrm{mg} / \mathrm{L})$

Table 7 Ceftaroline clinical cure rates by pathogen reported in Phase II and III clinical trials ${ }^{10,15,54}$

\begin{tabular}{|c|c|c|c|}
\hline \multirow[t]{2}{*}{ Pathogen } & \multirow{2}{*}{$\begin{array}{l}\text { Ceftaroline clinical cure rate \% } \\
\text { (CE population) } \\
\text { Phase II }\end{array}$} & \multicolumn{2}{|c|}{$\begin{array}{l}\text { Ceftaroline clinical cure rate \% } \\
\text { (ME population) }\end{array}$} \\
\hline & & CANVAS Ia & CANVAS $2^{\mathrm{a}}$ \\
\hline \multicolumn{4}{|l|}{ Gram-positive } \\
\hline Staphylococcus aureus & 96.7 & - & 93.3 \\
\hline MSSA & 100 & 91.3 & 94.4 \\
\hline MRSA & 80 & 95.1 & 91.4 \\
\hline Staphylococcus haemolyticus & 100 & - & - \\
\hline Streptococci & - & - & - \\
\hline S. pyogenes & 100 & 100 & 100 \\
\hline S. agalactiae & 100 & 93.8 & 100 \\
\hline Enterococcus faecalis & 100 & 92.9 & 63.6 \\
\hline Enterococcus faecium & 0 & - & - \\
\hline Group C streptococci & 100 & - & - \\
\hline Viridans group & 100 & - & - \\
\hline S. intermedius & 100 & - & 100 \\
\hline S. anginosus & 100 & - & 100 \\
\hline S. anginosus/"milleri" & 100 & - & - \\
\hline S. constellatus & - & - & 100 \\
\hline S. dysgalactiae & - & - & 100 \\
\hline S. oralis & 100 & - & - \\
\hline Peptostreptococcus prevotii & 100 & - & - \\
\hline Pediococcus spp & 100 & - & - \\
\hline Monomicrobial Gram-positive infections & - & - & 94.4 \\
\hline Polymicrobial Gram-positive infections & - & - & 92.3 \\
\hline \multicolumn{4}{|l|}{ Gram-negative } \\
\hline Enterobacteriaceae & - & - & 83.3 \\
\hline Escherichia coli & - & 90 & 100 \\
\hline Klebsiella pneumoniae & 100 & 90.9 & 100 \\
\hline Klebsiella oxytoca & - & - & 100 \\
\hline Enterobacter cloacae & 100 & - & 100 \\
\hline Proteus mirabilis & 0 & 70 & 60 \\
\hline Citrobacter freundii & 0 & - & - \\
\hline Morganella morganii & - & - & - \\
\hline Serratia marcescens & - & - & 100 \\
\hline Pseudomonas aeruginosa & - & - & 71.4 \\
\hline Monomicrobial Gram-negative infections & - & - & 100 \\
\hline Polymicrobial Gram-negative infections & - & - & 50 \\
\hline Mixed Gram-positive and Gram-negative infections & - & - & 88.9 \\
\hline
\end{tabular}

Note: aPhase III clinical trials.

Abbreviations: CE, clinically evaluable; ME, microbiologically evaluable; MSSA, methicillin-susceptible Staphylococcus aureus; MRSA, methicillin-resistant Staphylococcus aureus. 
Table 8 MIC $_{90}$ values reported for ceftaroline, vancomycin, and aztreonam activity against Gram-positive and Gram-negative pathogens commonly found in CSSSIs $10,14,15$

\begin{tabular}{|c|c|c|c|c|c|}
\hline \multirow[t]{2}{*}{ Pathogen } & \multicolumn{5}{|c|}{$M I C_{90}(\mathrm{mg} / \mathrm{L})$} \\
\hline & $\begin{array}{l}\text { Ceftaroline } \\
\text { CANVAS I }\end{array}$ & $\begin{array}{l}\text { Ceftaroline } \\
\text { CANVAS } 2\end{array}$ & $\begin{array}{l}\text { Ceftaroline } \\
\text { Jones et al }\end{array}$ & Vancomycin & Aztreonam \\
\hline Staphylococcus aureus (MSSA + MRSA) & 0.5 & 0.5 & 1.0 & 1.0 & - \\
\hline MSSA & 0.25 & 0.25 & $0.25-0.5$ & 1.0 & - \\
\hline MRSA & 1.0 & 0.5 & 1.0 & 1.0 & - \\
\hline CoNS & - & - & 0.5 & - & - \\
\hline Streptococcus pyogenes & $\leq 0.004$ & $\leq 0.004$ & - & 0.5 & - \\
\hline Streptococcus agalactiae & 0.015 & - & - & 0.5 & - \\
\hline Enterococcus faecalis & 8.0 & 1.0 & 2.0 & 2.0 & - \\
\hline$\beta$-hemolytic streptococci & - & - & $0.015-0.03$ & - & - \\
\hline Viridans streptococci & - & - & $0.012-0.25$ & - & - \\
\hline Escherichia coli & 1.0 & 0.5 & 0.25 to $>16$ & - & 0.12 \\
\hline Klebsiella pneumoniae & $>16$ & - & - & - & $>32$ \\
\hline Proteus mirabilis & $>16$ & NA & - & - & $\leq 0.03-0.25$ \\
\hline
\end{tabular}

Abbreviations: $\mathrm{MIC}_{90}$, minimum inhibitory concentration at which $90 \%$ of the isolates are inhibited; CoNS, coagulase-negative staphylococci; MSSA, methicillin-susceptible Staphylococcus aureus; MRSA, methicillin-resistant Staphylococcus aureus.

compared to vancomycin $(\leq 1.0 \mathrm{mg} / \mathrm{L}) \cdot{ }^{10,15}$ Ceftaroline also demonstrated inhibitory activity at a lower $\mathrm{MIC}_{90}$ against S. pyogenes and Streptococcus agalactiae versus vancomycin alone (Table 8). However, ceftaroline showed conflicting results against $E$. faecalis; it had a higher $\mathrm{MIC}_{90}$ against E. faecalis in CANVAS 1 yet demonstrated a lower $\mathrm{MIC}_{90}$ versus vancomycin alone in the Phase II and CANVAS 2 trials. ${ }^{10,14,15}$

A 2008 international surveillance study by Jones and colleagues evaluated ceftaroline activity against 14,169 isolates collected from cSSSI patients in the US and Europe. In US isolates, the study indicated ceftaroline activity against 2254 MRSA isolates $\left(\mathrm{MIC}_{90}, 1 \mathrm{mg} / \mathrm{L}\right)$. In European isolates, ceftaroline was active against $734 \mathrm{MRSA}$ isolates $\left(\mathrm{MIC}_{90}, 2 \mathrm{mg} / \mathrm{L}\right) .{ }^{14}$ The $\mathrm{MIC}_{90}$ for methicillin-susceptible $S$. aureus (MSSA) was $0.25-0.5 \mathrm{mg} / \mathrm{L}$. Ceftaroline was also active against coagulasenegative staphylococci $\left(\mathrm{MIC}_{90}, 0.5-1 \mathrm{mg} / \mathrm{L}\right)$, E. faecalis $\left(\mathrm{MIC}_{90}\right.$, $2 \mathrm{mg} / \mathrm{L}$ ), $\beta$-hemolytic streptococci $\left(\mathrm{MIC}_{90}, 0.015-0.03 \mathrm{mg} / \mathrm{L}\right.$ ), viridans group streptococci $\left(\mathrm{MIC}_{90}, 0.012-0.25 \mathrm{mg} / \mathrm{L}\right.$ ), and E. coli $\left(\mathrm{MIC}_{90}, 0.25\right.$ to $\left.>16 \mathrm{mg} / \mathrm{L}\right) .{ }^{14}$

Overall, in clinical trials ceftaroline proved efficacious in the treatment of cSSSIs, achieving high clinical cure rates and demonstrating high bactericidal activity against Grampositive and Gram-negative pathogens, including MRSA. Furthermore, ceftaroline demonstrated noninferiority to vancomycin plus aztreonam standard therapy. ${ }^{10,14,15}$

\section{Safety and tolerability}

Ceftaroline has a margin of safety and tolerability consistent with that of other cephalosporins. ${ }^{54}$ In the Phase II and CANVAS 1 and 2 clinical trials, ceftaroline demonstrated low or comparable incidence of adverse events to that of current standard therapy, with minimal or no effects on renal and hepatic function. ${ }^{10,15,54,57}$ The most common adverse events were nausea, diarrhea, headache, rash, and pruritis, with only $1.5 \%-6.5 \%$ of patients reporting any one of these symptoms during the course of therapy (Table 9). The majority $(70.8 \%-72.3 \%)$ of patients reported adverse events to be mild. ${ }^{10,15,54}$

An integrated safety summary of CANVAS 1 and 2 indicated only $28.3 \%$ of patients reported mild adverse symptoms, with $55.3 \%$ of patients reporting no symptoms at all. ${ }^{57}$ Elevated blood creatine phosphokinase was reported as a laboratory finding without clinically evident muscle or cardiac abnormalities. ${ }^{54}$ There was also no clinical evidence of hemolytic anemia. ${ }^{57}$ The percentage of patients with elevated alanine aminotransferase (ALT) or aspartate aminotransferase (AST) was low and not a cause for concern. ${ }^{54,57}$

A small percentage of patients $(4.1 \%-5.1 \%)$ developed a severe adverse event. An even smaller number of these patients discontinued treatment due to the severe adverse event. In the Phase II trial, all severe adverse events were resolved with no patient discontinuing treatment. ${ }^{10,15,54,57}$ The CANVAS 1 trial reported three deaths during the course of treatment; however, the causes of death were unrelated to the therapy or the cSSSI. ${ }^{10}$ Overall, the IV infusion of ceftaroline was well tolerated, with only $3 \%$ of patients reporting discomfort at the site of infusion in the Phase II trial. ${ }^{54}$

Integrated data from the CANVAS trials shows a smaller percentage of patients administered ceftaroline in comparison to vancomycin plus aztreonam experienced an increase in serum creatinine and blood urea nitrogen 
Table 9 Most common adverse events reported for ceftaroline $600 \mathrm{mg}$ IV every 12 hours for 5-I4 days treatment ${ }^{10,15,54,57}$

\begin{tabular}{|c|c|c|c|c|}
\hline & \multirow{2}{*}{$\begin{array}{l}\text { \% of patients (AE) } \\
\text { Phase II }\end{array}$} & \multicolumn{3}{|c|}{$\%$ of patients (TEAE) } \\
\hline & & CANVAS I & CANVAS 2 & $\begin{array}{l}\text { Integrated CANVAS } \\
\text { summary }\end{array}$ \\
\hline \multicolumn{5}{|l|}{ Most common } \\
\hline Nausea & 6.0 & 5.7 & 6.2 & 5.9 \\
\hline Diarrhea & - & 3.4 & 6.5 & 4.9 \\
\hline Headache & 6.0 & 5.1 & 5.3 & 5.2 \\
\hline Rash & 1.5 & - & - & 3.2 \\
\hline Pruritis & - & 3.1 & 3.8 & 3.5 \\
\hline Elevated blood CPK & 7.5 & - & - & - \\
\hline Crystals in urine & 9.0 & - & - & - \\
\hline Elevated ALT & 6.0 & - & - & 1.2 \\
\hline Elevated AST & 6.0 & - & - & 1.0 \\
\hline Patients with a SAE & 5.1 & 4.6 & 4.1 & 4.3 \\
\hline Patients who died during study & 0 & 0.9 & 0 & 0 \\
\hline $\begin{array}{l}\text { Patients discontinued due } \\
\text { to } A E \text { or TEAE }\end{array}$ & 3.0 & 3.7 & 2.3 & 3.0 \\
\hline Infusion site erythema/swelling & 3.1 & - & - & 1.0 \\
\hline \multicolumn{5}{|l|}{ Renal abnormalities } \\
\hline $\begin{array}{l}\text { Serum creatinine }>1.5 \mathrm{mg} / \mathrm{dL} \\
\text { and }>50 \% \text { increase }\end{array}$ & - & - & - & 0.9 \\
\hline BUN $>1.5 X$ ULN and $>50 \%$ increase & - & - & - & 0.3 \\
\hline Creatinine clearance $>50 \%$ increase & - & - & - & 0.4 \\
\hline \multicolumn{5}{|l|}{ Liver function abnormalities } \\
\hline $\begin{array}{l}\text { ALT or AST }>3 \times \text { ULN, ALP } \\
<2 \times \text { ULN and total bilirubin } \\
>2 \times \text { ULN }\end{array}$ & - & - & - & 0 \\
\hline Positive direct Coombs' test & - & $\begin{array}{l}\text { Ceftaroline } \\
\text { Group }>\text { standard } \\
\text { therapy group }\end{array}$ & $\begin{array}{l}\text { Ceftaroline } \\
\text { Group }>\text { standard } \\
\text { therapy group }\end{array}$ & 11.6 \\
\hline
\end{tabular}

Abbreviations: AE, adverse event; CPK, creatine phosphokinase; ALT, alanine aminotransferase; AST, aspartate aminotransferase; SAE, severe adverse event; TEAE, treatment-emergent adverse event; BUN, blood urea nitrogen; ULN, upper limit of normal; ALP, alkaline phosphatase.

( $0.9 \%$ and $0.3 \%$ vs $2.1 \%$ and $1.2 \%$, respectively) demonstrating that ceftaroline has less impact on renal function compared to standard treatment. ${ }^{57}$ One patient developed acute renal failure, however, it was unclear whether the event occurred as a result of the treatment. ${ }^{57}$ Potential for hepatic damage was also evaluated. In both the ceftaroline and vancomycin plus aztreonam groups, no patients had an increase in ALT or AST greater than threefold above the upper limit of normal, less than twofold increase in alkaline phosphatase above the upper limit of normal, or a greater than twofold increase in bilirubin above the upper limit of normal; thus, no hepatic damage was noted in any patient. ${ }^{57}$

In the CANVAS trials, three patients in the ceftaroline group were reported to have Clostridium difficile gastrointestinal infection versus only one in the vancomycin plus aztreonam groups.$^{57}$ In the Phase II trial, no patients discontinued treatment due to noncompliance or unsatisfactory therapeutic response. ${ }^{54}$ In CANVAS 1, only one patient discontinued treatment due to noncompliance and no patients discontinued due to lack of clinical progress. ${ }^{10}$ Similarly in CANVAS 2, no patients discontinued treatment due to noncompliance, however, two patients discontinued due to lack of clinical progress. ${ }^{15}$

In summary, clinical trial data demonstrated ceftaroline is generally safe and well tolerated in the treatment of cSSSIs. The number of patients reporting adverse events related to the course of therapy was low, with the majority of these adverse events being mild. There was also minimal or no effect on renal and hepatic function, making ceftaroline a safe option in the treatment of cSSSIs.

\section{Advantages and disadvantages of ceftaroline in cSSSIs Problems with current cSSSls therapeutics}

The emergence of vancomycin resistance among bacterial pathogens associated with cSSSIs is a growing concern in the treatment of these infections. ${ }^{41}$ Vancomycin also carries the risk for nephrotoxicity as well as development of red-man syndrome when administered too rapidly. ${ }^{72,73}$ Disadvantages of linezolid 
include bacteriostatic activity (rather than bactericidal) against staphylococci and enterococci, potential toxicity with prolonged therapy, myelosuppression, and monoamine oxidase inhibition. ${ }^{3,74}$ Daptomycin lacks activity against Gram-negative pathogens, can cause muscular toxicity, and must be used with caution in patients taking statins. ${ }^{74,75}$ Similarly, telavancin lacks activity against Gram-negative pathogens. ${ }^{76}$ Telavancin should also be avoided in patients with cardiac disease and those who undergo routine coagulation tests, since telavancin produces false-positive blood test abnormalities. ${ }^{42}$ Tigecycline is only bacteriostatic, can cause digestive system side effects, tooth discoloration, acute pancreatitis, and is contraindicated in pregnant women., ${ }^{877}$ Tigecycline must also be used with caution in patients with hepatic impairment or intestinal perforation. $^{78}$

\section{Advantages of ceftaroline in cSSSls}

Ceftaroline is the first cephalosporin with a high clinical cure rate for cSSSIs caused by CA-MRSA and HA-MRSA. 10,14,15,47,53,54 It has broad-spectrum bactericidal activity with high activity against many opportunistic pathogens. Clinical trials reported that the most commonly encountered adverse events were mild, with very few patients experiencing swelling or pain at the infusion site. ${ }^{10,15,54}$ Further, few patients experienced renal or hepatic abnormalities related to ceftaroline therapy, and thus, ceftaroline is recommended for use in patients with renal impairment at adjusted doses. Although the pharmacokinetics of ceftaroline in patients with hepatic impairment have not been established, its minimal hepatic metabolism suggests systemic clearance of the drug is not significantly affected by hepatic impairment. Additionally, ceftaroline demonstrated very few clinically evident muscle or cardiac abnormalities. ${ }^{10,15,54}$

\section{Disadvantages of ceftaroline in cSSSIs}

There is no consensus on ceftaroline's activity against certain extended-spectrum $\beta$-lactamase-producing (ESBL) strains. Biek and colleagues reported that ceftaroline is inactive against ESBL-producing Enterobacteriaceae. ${ }^{79}$ A different study, however, demonstrated that ceftaroline does possess activity against ESBL-producing $E$. coli and $K$. pneumoniae strains at high concentrations $\left(\mathrm{MIC}_{90}\right.$ of $\left.\geq 32 \mu \mathrm{g} / \mathrm{mL}\right) .{ }^{80}$ Similarly, another study showed ceftaroline to be active at high MICs against ESBL-producing strains regardless of species. ${ }^{81}$ Most in vitro studies have also shown that ceftaroline has only limited activity against $P$. aeruginosa..$^{42,79-81}$ However in the CANVAS 2 clinical trial, ceftaroline demonstrated a high clinical cure rate against $P$. aeruginosa in five of seven $\left(71.4 \%\right.$ ) patients in the ME population. ${ }^{15}$
Additionally, there are no well-controlled clinical trials evaluating ceftaroline use in pregnant women, and thus, its use in pregnancy should only occur if the benefit justifies the potential risk to the fetus. The safety and effectiveness of ceftaroline in the pediatric population, patients receiving systemic corticosteroid therapy, patients with HIV, and patients with a recent history of chemotherapy or radiation therapy has also not been established. ${ }^{15,64}$

\section{Patient considerations}

Although ceftaroline has demonstrated a high clinical cure rate in cSSSIs, especially when MRSA strains are involved, to date there are no data available regarding patient compliance and satisfaction during ceftaroline therapy or quality of life following treatment. In the Phase II and CANVAS trials, a large percentage of patients presented with severe signs and symptoms at baseline, which included fever, major abscesses, extensive cellulitis, or complicating factors such as diabetes mellitus and peripheral vascular disease. ${ }^{10,15,54} \mathrm{~A}$ severe clinical presentation and the need for hospital-based treatment could be determinants leading to high patient compliance and satisfaction; however, future studies are necessary to examine these patient-related factors and the impact of ceftaroline treatment on quality of life.

The cost of ceftaroline is US\$41 for either a $400 \mathrm{mg}$ or $600 \mathrm{mg}$ vial. The total cost of ceftaroline treatment for a patient with normal renal function is US\$82/day or US\$574/7-day course of treatment. ${ }^{58}$

\section{Conclusion}

Ceftaroline is a potent, safe, and effective new cephalosporin for the treatment of cSSSIs, including those caused by CAMRSA and HA-MRSA. Because of its broad-spectrum coverage, bactericidal activity, tolerability, and minimal side effect profile, ceftaroline is an attractive first-line therapeutic choice for the treatment of cSSSIs, including infections in which MRSA is suspected.

\section{Disclosure}

The authors declare no conflicts of interest in this work.

\section{References}

1. FDA.gov [homepage on the Internet]. Maryland: Guidance for industry: Uncomplicated and complicated skin and skin structure infections: developing antimicrobial drugs for treatment; [cited Jul 1998]. Available from: http://www.fda.gov/ohrms/dockets/ac/03/briefing/3997B1_02_GFIDiabetic\%20Foot.pdf. Accessed September 26, 2011.

2. FDA.gov [homepage on the Internet]. Maryland: Guidance for industry: Acute bacterial skin and skin structure infections: developing drugs for treatment; [cited Aug 2010]. Available from: http://www.fda. gov/downloads/Drugs/GuidanceComplianceRegulatoryInformation/ Guidances/ucm071185.pdf. Accessed September 26, 2011. 
3. Nathwani D. New antibiotics for the management of complicated skin and soft tissue infections: are they any better? Int J Antimicrob Agents. 2009;34 Suppl 1:S24-S29.

4. Liu C, Bayer A, Cosgrove SE, et al. Clinical practice guidelines by the Infectious Diseases Society of America for the treatment of methicillinresistant Staphylococcus aureus infections in adults and children: executive summary. Clin Infect Dis. 2011;52(3):285-292.

5. DiNubile MJ, Lipsky BA. Complicated infections of skin and skin structures: when the infection is more than skin deep. J Antimicrob Chemother. 2004;53 Suppl 2:ii37-ii50.

6. Lee SY, Kuti JL, Nicolau DP. Antimicrobial management of complicated skin and skin structure infections in the era of emerging resistance. Surg Infect (Larchmt). 2005;6(3):283-295.

7. Grolman DC. Therapeutic applications of tigecycline in the management of complicated skin and skin structure infections. Int J Infect Dis. 2007;11 Suppl 1:S7-S15.

8. Reygaert WC. Antibiotic optimization in the difficult-to-treat patient with complicated intra-abdominal or complicated skin and skin structure infections: focus on tigecycline. Ther Clin Risk Manag. 2010;6:419-430.

9. Barbour A, Derendorf H. Resistance and the management of complicated skin and skin structure infections: the role of ceftobiprole. Ther Clin Risk Manag. 2010;6:485-495.

10. Corey GR, Wilcox MH, Talbot GH, Thye D, Friedland D, Baculik T. CANVAS 1: the first Phase III, randomized, double-blind study evaluating ceftaroline fosamil for the treatment of patients with complicated skin and skin structure infections. J Antimicrob Chemother. 2010; 65 Suppl 4:iv41-iv51.

11. Fung HB, Chang JY, Kuczynski S. A practical guide to the treatment of complicated skin and soft tissue infections. Drugs. 2003;63(14): 1459-1480.

12. Goldstein EJ, Citron DM, Merriam CV, Warren Y, Tyrrell KL, Gesser RM. General microbiology and in vitro susceptibility of anaerobes isolated from complicated skin and skin-structure infections in patients enrolled in a comparative trial of ertapenem versus piperacillin-tazobactam. Clin Infect Dis. 2002;35 Suppl 1:S119-S125.

13. Graham DR, Lucasti C, Malafaia O, et al. Ertapenem once daily versus piperacillin-tazobactam 4 times per day for treatment of complicated skin and skin-structure infections in adults: results of a prospective, randomized, double-blind multicenter study. Clin Infect Dis. 2002;34(11):1460-1468.

14. Jones RN, Mendes RE, Sader HS. Ceftaroline activity against pathogens associated with complicated skin and skin structure infections: results from an international surveillance study. J Antimicrob Chemother. 2010;65 Suppl 4:iv17-iv31.

15. Wilcox MH, Corey GR, Talbot GH, Thye D, Friedland D, Baculik T. CANVAS 2: the second Phase III, randomized, double-blind study evaluating ceftaroline fosamil for the treatment of patients with complicated skin and skin structure infections. J Antimicrob Chemother. 2010;65 Suppl 4:iv53-iv65.

16. Moran GJ, Krishnadasan A, Gorwitz RJ, et al. Methicillin-resistant $S$. aureus infections among patients in the emergency department. N Engl J Med. 2006;355(7):666-674.

17. Stein GE, Wells EM. The importance of tissue penetration in achieving successful antimicrobial treatment of nosocomial pneumonia and complicated skin and soft-tissue infections caused by methicillin-resistant Staphylococcus aureus: vancomycin and linezolid. Curr Med Res Opin. 2010;26(3):571-588.

18. King MD, Humphrey BJ, Wang YF, Kourbatova EV, Ray SM, Blumberg HM. Emergence of community-acquired methicillin-resistant Staphylococcus aureus USA300 clone as the predominant cause of skin and soft-tissue infections. Ann Intern Med. 2006;144(5):309-317.

19. Suzuki. Antimicrobial ointments and methicillin-resistant Staphylococcus aureus USA300. Emerg Infect Dis. 2011;17(10):1917-1920.

20. Micek ST, Hoban AP, Pham V, Doherty JA, Kollef MH. Institutional perspective on the impact of positive blood cultures on the economic and clinical outcomes of patients with complicated skin and skin structure infections: Focus on Gram-positive infections. Clin Ther. 2011;33(11):1759-1768.
21. Hatoum HT, Akhras KS, Lin SJ. The attributable clinical and economic burden of skin and skin structure infections in hospitalized patients: a matched cohort study. Diagn Microbiol Infect Dis. 2009;64(3):305-310.

22. Edelsberg J, Berger A, Weber DJ, Mallick R, Kuznik A, Oster G. Clinical and economic consequences of failure of initial antibiotic therapy for hospitalized patients with complicated skin and skin-structure infections. Infect Control Hosp Epidemiol. 2008;29(2):160-169.

23. Mendoza N, Tyring SK. Emerging drugs for complicated skin and skinstructure infections. Expert Opin Emerg Drugs. 2010;15(3):509-520.

24. Napolitano LM. Early appropriate parenteral antimicrobial treatment of complicated skin and soft tissue infections caused by methicillin-resistant Staphylococcus aureus. Surg Infect (Larchmt). 2008;9 Supp1 1:s17-s27.

25. Grayson ML. The treatment triangle for staphylococcal infections. N Engl J Med. 2006;355(7):724-727.

26. Culos KA, Cannon JP, Grim SA. Alternative agents to vancomycin for the treatment of methicillin-resistant Staphylococcus aureus infections. Am J Ther. October 17, 2011; 45 Suppl 3:S184-190.

27. Moellering RC Jr. The problem of complicated skin and skin structure infections: the need for new agents. J Antimicrob Chemother. 2010; 65 Suppl 4:iv3-iv8.

28. Raghavan M, Linden PK. Newer treatment options for skin and soft tissue infections. Drugs. 2004;64(15):1621-1642.

29. Kraus CN, Burnstead BW. The safety record of fusidic acid in non-US markets: a focus on skin infections. Clin Infect Dis. 2011;52 Suppl 7: S527-S537.

30. Craft JC, Moriarty SR, Clark K, et al. A randomized, double-blind phase 2 study comparing the efficacy and safety of an oral fusidic acid loading-dose regimen to oral linezolid for the treatment of acute bacterial skin and skin structure infections. Clin Infect Dis. 2011;52 Suppl 7: S520-S526.

31. Cercenado E. Update of antimicrobial resistance in Gram-positive microorganisms. Med Clin (Barc). 2010;135 Suppl 3:10-15.

32. Mera RM, Suaya JA, Amrine-Madsen H, et al. Increasing role of Staphylococcus aureus and community-acquired methicillinresistant Staphylococcus aureus infections in the United States: a 10-year trend of replacement and expansion. Microb Drug Resist. 2011;17(2):321-328.

33. Nichol KA, Adam HJ, Hussain Z, et al. Comparison of communityassociated and health care-associated methicillin-resistant Staphylococcus aureus in Canada: results of the CANWARD 2007-2009 study. Diagn Microbiol Infect Dis. 2011;69(3):320-325.

34. National Nosocomial Infections Surveillance System. National Nosocomial Infections Surveillance (NNIS) System Report, data summary from January 1992 through June 2004, issued October 2004. Am J Infect Control. 2004;32(8):470-485.

35. Tattevin P, Diep BA, Jula M, Perdreau-Remington F. Methicillinresistant Staphylococcus aureus USA300 clone in long-term care facility. Emerg Infect Dis. 2009;15(6):953-955.

36. Crum NF, Lee RU, Thornton SA, et al. Fifteen-year study of the changing epidemiology of methicillin-resistant Staphylococcus aureus. Am J Med. 2006;119(11):943-951.

37. Krziwanek K, Metz-Gercek S, Mittermayer H. Trends in the occurrence of MRSA strains in Upper Austria from 2006 to 2009. Clin Microbiol Infect. 2011;17(6):920-923.

38. Elston JW, Barlow GD. Community-associated MRSA in the United Kingdom. J Infect. 2009;59(3):149-155.

39. Goud R, Gupta S, Neogi U, et al. Community prevalence of methicillin and vancomycin resistant Staphylococcus aureus in and around Bangalore, southern India. Rev Soc Bras Med Trop. 2011;44(3):309-312.

40. CDC.gov [homepage on the Internet]. Atlanta: MRSA Infections. [Updated April 8, 2011] Available from: http://www.cdc.gov/mrsa/ statistics/index.html. Accessed September 29, 2011.

41. Sader HS, Jones RN. Antimicrobial susceptibility of Gram-positive bacteria isolated from US medical centers: results of the Daptomycin Surveillance Program (2007-2008). Diagn Microbiol Infect Dis. 2009;65(2):158-162. 
42. Nailor MD, Sobel JD. Antibiotics for Gram-positive bacterial infection: vancomycin, teicoplanin, quinupristin/dalfopristin, oxazolidinones, daptomycin, telavancin, and ceftaroline. Med Clin North Am. 2011;95(4):723-742, vii.

43. Sader HS, Farrell DJ, Jones RN. Antimicrobial susceptibility of Grampositive cocci isolated from skin and skin-structure infections in European medical centres. Int J Antimicrob Agents. 2010;36(1):28-32.

44. Van Hal SJ, Paterson DL. Systematic review and meta-analysis of the significance of heterogeneous vancomycin-intermediate Staphylococcus aureus isolates. Antimicrob Agents Chemother. 2011;55(1): 405-410.

45. FDA.gov [homepage on the internet]. Maryland: Teflaro label and approval history. [Updated daily, cited Nov 2010]. Available from: http:// www.accessdata.fda.gov/drugsatfda-docs/label/2011/200237s001lbl. pdf. Accessed September 30, 2011.

46. Fenoll A, Aguilar L, Robledo O, et al. In vitro activity of ceftaroline against Streptococcus pneumoniae isolates exhibiting resistance to penicillin, amoxicillin, and cefotaxime. Antimicrob Agents Chemother. 2008; 52(11):4209-4210.

47. Ge Y, Biek D, Talbot GH, Sahm DF. In vitro profiling of ceftaroline against a collection of recent bacterial clinical isolates from across the United States. Antimicrob Agents Chemother. 2008;52(9):3398-3407.

48. Jacqueline C, Caillon J, Le Mabecque V, et al. In vivo activity of a novel anti-methicillin-resistant Staphylococcus aureus cephalosporin, ceftaroline, against vancomycin-susceptible and -resistant Enterococcus faecalis strains in a rabbit endocarditis model: a comparative study with linezolid and vancomycin. Antimicrob Agents Chemother. 2009;53(12):5300-5302.

49. Jacqueline C, Caillon J, Le Mabecque V, et al. In vivo efficacy of ceftaroline (PPI-0903), a new broad-spectrum cephalosporin, compared with linezolid and vancomycin against methicillin-resistant and vancomycin-intermediate Staphylococcus aureus in a rabbit endocarditis model. Antimicrob Agents Chemother. 2007;51(9):3397-3400.

50. McGee L, Biek D, Ge Y, et al. In vitro evaluation of the antimicrobial activity of ceftaroline against cephalosporin-resistant isolates of Streptococcus pneumoniae. Antimicrob Agents Chemother. 2009;53(2): $552-556$.

51. Morrissey I, Ge Y, Janes R. Activity of the new cephalosporin ceftaroline against bacteraemia isolates from patients with community-acquired pneumonia. Int J Antimicrob Agents. 2009;33(6):515-519.

52. Mushtaq S, Warner M, Ge Y, Kaniga K, Livermore DM. In vitro activity of ceftaroline (PPI-0903M, T-91825) against bacteria with defined resistance mechanisms and phenotypes. J Antimicrob Chemother. 2007;60(2):300-311.

53. Sader HS, Fritsche TR, Jones RN. Antimicrobial activities of ceftaroline and ME1036 tested against clinical strains of community-acquired methicillin-resistant Staphylococcus aureus. Antimicrob Agents Chemother. 2008;52(3):1153-1155.

54. Talbot GH, Thye D, Das A, Ge Y. Phase 2 study of ceftaroline versus standard therapy in treatment of complicated skin and skin structure infections. Antimicrob Agents Chemother. 2007;51(10):3612-3616.

55. Vidaillac C, Leonard SN, Sader HS, Jones RN, Rybak MJ. In vitro activity of ceftaroline alone and in combination against clinical isolates of resistant gram-negative pathogens, including beta-lactamase-producing Enterobacteriaceae and Pseudomonas aeruginosa. Antimicrob Agents Chemother. 2009;53(6):2360-2366.

56. Corey GR, Wilcox M, Talbot GH, et al. Integrated analysis of CANVAS 1 and 2: phase 3 , multicenter, randomized, double-blind studies to evaluate the safety and efficacy of ceftaroline versus vancomycin plus aztreonam in complicated skin and skin-structure infection. Clin Infect Dis. 2010;51(6):641-650.

57. Corrado ML. Integrated safety summary of CANVAS 1 and 2 trials: Phase III, randomized, double-blind studies evaluating ceftaroline fosamil for the treatment of patients with complicated skin and skin structure infections. J Antimicrob Chemother. 2010; 65 Suppl 4:iv67-iv71.
58. Beckwith MC, Vu J, editors. New Drug Bulletin: Ceftaroline Fosamil (Teflaro $^{\mathrm{TM}}$-Forest Pharmaceuticals). Salt Lake City: University of Utah Hospitals and Clinics; 2011.

59. Kaushik D, Rathi S, Jain A. Ceftaroline: a comprehensive update. Int J Antimicrob Agents. 2011;37(5):389-395.

60. Saravolatz LD, Stein GE, Johnson LB. Ceftaroline: a novel cephalosporin with activity against methicillin-resistant Staphylococcus aureus. Clin Infect Dis. 2011;52(9):1156-1163.

61. Drusano GL. Pharmacodynamics of ceftaroline fosamil for complicated skin and skin structure infection: rationale for improved antimethicillin-resistant Staphylococcus aureus activity. J Antimicrob Chemother. 2010;65 Suppl 4:iv33-iv39.

62. Zhanel GG, Sniezek G, Schweizer F, et al. Ceftaroline: a novel broadspectrum cephalosporin with activity against meticillin-resistant Staphylococcus aureus. Drugs. 2009;69(7):809-831.

63. Lim L, Sutton E, Brown J. Ceftaroline: a new broad-spectrum cephalosporin. Am J Health Syst Pharm. 2011;68(6):491-498.

64. Teflaro.com [homepage on the Internet]. St Louis: Teflaro: proven effective in acute bacterial skin and skin structure infections (ABSSSI) caused by susceptible bacteria. Highlights of prescribing information. [Updated Apr 2011] Available from: http://www.frx.com/pi/teflaro_ pi.pdf. Accessed September 30, 2011

65. Steed ME, Rybak MJ. Ceftaroline: a new cephalosporin with activity against resistant gram-positive pathogens. Pharmacotherapy. 2010;30(4):375-389.

66. Riccobene T, Fang E, Thye D. A single- and multiple-dose study to determine the safety, tolerability, and pharmacokinetics (PK) of ceftaroline (CPT) administered by intramuscular (IM) injection to healthy subjects [abstract no A-1888]. Proceedings of the 48th Interscience Conference on Antimicrobial Agents and Chemotherapy/46th Infectious Diseases Society of America. October 25-28, 2008. Washington, DC.

67. Ge Y, Thye D, Liao S, Talbot GH. Pharmacokinetics (PK) of ceftaroline (PPI0903) in subjects with mild or moderate renal impairment (RI) [abstract no A-1939]. Proceedings of the 46th Interscience Conference on Antimicrobial Agents and Chemotherapy; September 27-30, 2006. San Francisco, CA.

68. Riccobene T, Jakate A, Rank D, Thye DA. An open-label, pharmacokinetic, safety and tolerability study of single-dose intravenous ceftaroline in subjects with end-stage renal disease on intermittent haemodialysis. Poster presentation at the European congress of clinical microbiology and infectious diseases, Helsinki, Finland. May 16-19, 2009.

69. Andes D, Craig WA. Pharmacodynamics of a new cephalosporin, PPI0903 (TAK-599), active against methicillin-resistant Staphylococcus aureus in murine thigh and lung infection models: identification of an in vivo pharmacokinetic-pharmacodynamic target. Antimicrob Agents Chemother. 2006;50(4):1376-1383.

70. Levinson W. Medical Microbiology and Immunology. 10th ed. San Francisco, CA: McGraw-Hill; 2008.

71. Vidaillac C, Leonard SN, Rybak MJ. In vitro activity of ceftaroline against methicillin-resistant Staphylococcus aureus and heterogeneous vancomycin-intermediate S. aureus in a hollow fiber model. Antimicrob Agents Chemother. 2009;53(11):4712-4717.

72. Lodise TP, Lomaestro B, Graves J, Drusano GL. Larger vancomycin doses (at least four grams per day) are associated with an increased incidence of nephrotoxicity. Antimicrob Agents Chemother. 2008;52(4):1330-1336.

73. Polk RE, Healy DP, Schwartz LB, Rock DT, Garson ML, Roller K. Vancomycin and the red-man syndrome: pharmacodynamics of histamine release. J Infect Dis. 1988;157(3):502-507.

74. Powell JP, Wenzel RP. Antibiotic options for treating communityacquired MRSA. Expert Rev Anti Infect Ther. 2008;6(3):299-307.

75. Arbeit RD, Maki D, Tally FP, Campanaro E, Eisenstein BI. The safety and efficacy of daptomycin for the treatment of complicated skin and skin-structure infections. Clin Infect Dis. 2004;38(12):1673-1681.

76. Rubinstein E, Lalani T, Corey GR, et al. Telavancin versus vancomycin for hospital-acquired pneumonia due to Gram-positive pathogens. Clin Infect Dis. 2011;52(1):31-40. 
77. Ellis-Grosse EJ, Babinchak T, Dartois N, Rose G, Loh E. The efficacy and safety of tigecycline in the treatment of skin and skinstructure infections: results of 2 double-blind phase 3 comparison studies with vancomycin-aztreonam. Clin Infect Dis. 2005;41 Suppl 5: S341-S353.

78. Tygacil [homepage on the Internet]. New York: Expanded broadspecrum IV antibiotic indicated for adults with cSSSI, cIAI, and CABP due to the indicated pathogens. Available from: http://www.pfizerpro. com/hcp/tygacil/indications/cSSSI. Accessed October 5, 2011.

79. Biek D, Critchley IA, Riccobene TA, Thye DA. Ceftaroline fosamil: a novel broad-spectrum cephalosporin with expanded anti-Gram-positive activity. J Antimicrob Chemother. 2010;65 Suppl 4:iv9-iv16.
80. Brown SD, Traczewski MM. In vitro antimicrobial activity of a new cephalosporin, ceftaroline, and determination of quality control ranges for MIC testing. Antimicrob Agents Chemother. 2009;53(3): 1271-1274.

81. Sader HS, Fritsche TR, Kaniga K, Ge Y, Jones RN. Antimicrobial activity and spectrum of PPI-0903M (T-91825), a novel cephalosporin, tested against a worldwide collection of clinical strains. Antimicrob Agents Chemother. 2005;49(8):3501-3512.

\section{Publish your work in this journal}

Infection and Drug Resistance is an international, peer-reviewed openaccess journal that focuses on the optimal treatment of infection (bacterial, fungal and viral) and the development and institution of preventive strategies to minimize the development and spread of resistance. The journal is specifically concerned with the epidemiology of antibiotic

\section{Dovepress}

resistance and the mechanisms of resistance development and diffusion in both hospitals and the community. The manuscript management system is completely online and includes a very quick and fair peerreview system, which is all easy to use. Visit http://www.dovepress.com/ testimonials.php to read real quotes from published authors.

Submit your manuscript here: http://www.dovepress.com/infection-and-drug-resistance-journal 